\title{
Evaluación de la tasa de concepción de tres razas bovinas receptoras de embriones en el Trópico Alto
}

\author{
Conception Rate Evaluation of Three Different Breeds's \\ Recipients of Bovine Embryos in High Tropical Lands
}

Fecha recepción: 6 de mayo de 2013

Fecha Aprobación: 28 de noviembre de 2013

Oscar Orlando Farfan-Rojas¹, José Luis Porras-Vargas²

\section{RESUMEN}

El presente estudio se realizó con el fin de evaluar la eficiencia reproductiva, medida en porcentaje de novillas preñadas, de tres razas bovinas, como receptoras de embriones. En el altiplano Cundiboyacense se han realizado pocos estudios tendientes a determinar cuáles razas, aptas para el medio, son las que pueden brindar mejores tasas de concepción, como receptoras, en transferencia de embriones. Los resultados se sometieron a un análisis estadístico descriptivo, utilizando los programas Excel y Minitab (Chi cuadrado x²), con variables de tipo cuantitativo. La selección de receptoras se hizo con base en la calidad de cuerpo lúteo, normalidad del tracto reproductivo, CC entre 2 y 3 en una escala de 1 (muy flaca) a 5 (cebada). La transferencia de embriones se realizó por el método no quirúrgico y se obtuvo un porcentaje de gestación en la raza Normando de $63 \%$; en la Holstein, de $55.5 \%$, y en la raza Brangus, de $44.4 \%$. El protocolo utilizado fue

\begin{abstract}
The present study was conducted to evaluate the reproductive efficiency on heifers pregnancy rates from three different breeds, as embryo recipients. In the Cundiboyacense highlands, there has been few studies designed to determine which breeds beside, suitable for the environment, are the ones that can provide the best pregnancy rates, as embryo transfer recipients. The results were studied by descriptive statistical analysis using Excel and Minitab (Chi square $x^{2}$ ) programs, with quantitative variables. The selection of recipients was based on the quality of the corpus luteum, CC normal reproductive tract, between 2 and 3 on a scale from 1 (very weak) to 5 (fat). The embryo transfer was performed by a non-surgically method: The pregnancy rates found were: for Normando Breed of $63 \%$, Holstein, $55.5 \%$ and $44.4 \%$ for Brangus. The protocol used was Ovsynch, and the
\end{abstract}

Profesional Independiente. Correo electrónico: oscarorlando08@hotmail.com

M.Sc. Universidad Pedagógica y Tecnológica de Colombia (Tunja, Boyacá-Colombia). Correo electrónico: jose.vargas@ uptc.edu.co 
Ovisynch, y los embriones transferidos fueron congelados y descongelados para el momento de transferirlos.

Palabras clave: Normando, Holstein, Brangus, Novillas receptoras, Transferencia de embriones, Trópico alto. transferred embryos were frozen and thawed at the time of transfer.

Key Words: Norman, Holstein, Brangus, Recipients heifers, Embryo transfer, Tropic High Land. 


\section{INTRODUCCIÓN}

El éxito de un programa masivo de transferencia de embriones (TE) depende de varias variables; en general, las receptoras deben ser animales mansos, de buenas características reproductivas, con buen instinto materno, buena capacidad lechera, facilidad de parto y adaptabilidad al medio, entre otros.

En el altiplano Cundiboyacense se han realizado pocos estudios tendientes a determinar cuál de las razas, aptas para el medio, puede brindar mejores tasas de concepción, como receptoras de embriones. Para acelerar el mejoramiento de nuestra ganadería, tanto de carne como de leche, se puede utilizar como herramienta la TE, que permite disminuir el tiempo generacional para mejorar genéticamente las ganaderías; pero hay que tener en cuenta que las tasas de novillas preñadas en transferencia de embriones congelados o frescos en el país son bajas, y los costos son altos (1).

Según Hansen (1), el éxito de estos programas depende de diversas variables, entre ellas: calidad del embrión, experiencia del técnico y nutrición de las receptoras de los embriones, las cuales, dependiendo de diversos factores, le brindará, en menor o mayor porcentaje, la supervivencia y adaptabilidad al embrión, primero a nivel uterino $y$, luego, si se concibe la preñez, al medioambiente exterior.

Estas son las razones por las cuales se hace necesario adelantar estudios relacionados con la selección de receptoras de embriones en la región, que cuenta con razas Bostaurus adaptadas (Holstein, Normando, Brangus); de estas pretendemos, en este estudio, determinar cuál brinda mejores tasas de eficiencia reproductiva como receptora, para así orientar a quien decida llevar a cabo programas de transferencia de embriones en el trópico alto.

El objetivo del presente trabajo fue evaluar la tasa de concepción de receptoras de embriones de diferentes razas bovinas, en el trópico alto, y establecer parámetros para la selección de estas, a partir de las ventajas y las desventajas entre las tres razas de receptoras de embriones en estudio.

\section{MATERIALES Y MÉTODOS}

El estudio se realizó en la finca Cid Campeador Ventaquemada, del Centro Internacional de Biotecnología Reproductiva (CIBre); localizada en el municipio de Ventaquemada (Boyacá), que pertenece a la región geográfica Andina y se ubica en el altiplano Cundí-boyacense. La altitud aproximada de la finca está entre los 2725 y 2825 msnm. En Ventaquemada predomina el clima frío húmedo; la temperatura promedio es de $14{ }^{\circ} \mathrm{C}$; el promedio anual de precipitación es de $1613 \mathrm{~mm}$, y la humedad relativa, de $83.5 \%$.

El trabajo de campo se llevó a cabo con 298 hembras bovinas de las razas Normando (88), Holstein (108) y Brangus (102). Se seleccionaron las novillas cíclicas y reproductivamente sanas, mediante examen ginecológico (palpación rectal y ecografía), condición corporal igual o superior a 2.8 (en escala de 0 a 5), según clasificación para ganado de leche o ganado de carne, con peso vivo mínimo de $350 \mathrm{~kg}$. Esta selección se realizó con el fin de determinar las hembras que fueron sometidas al protocolo de sincronización de celo. A los 7 días posteriores al celo, el técnico realizó la selección, mediante palpación rectal, de hembras aptas para recibir el embrión según su criterio y relación (cuerpo lúteo mayor de 16 $\mathrm{mm}, \mathrm{CC}$ mayor o igual a 2.8 y animales de buena conformación corporal).

Los resultados se analizaron mediante un análisis estadístico descriptivo, con variables de tipo cuantitativo y con el ánimo de determinar el efecto de estas variables sobre los porcentajes de preñez obtenidos; dichas variables evaluadas fueron: el porcentaje de novillas gestantes de cada raza; la raza (grupo genético) y la condición corporal y peso.

\section{RESULTADOS Y DISCUSIÓN}

El porcentaje general de novillas preñadas fue de $37.12 \%$ (preñadas/disponibles) y $51.1 \%$ (preñadas/ transferidas); este resultado está dentro de los parámetros de Gómez (2) en Colombia (50\%), es similar al de De Luca (3) en Argentina (51.45\%) y es superior al de Mato Groso, Brasil, (10.4\%) y 
$(39.4 \%)$, y al de Santa Cruz Bolivia, $(15.9 \%)$ y $(49.8 \%)$, pero lejos aún de los niveles de USA y Europa $(80 \%)$.

Tabla I. Resultados generales

\begin{tabular}{|c|c|c|c|c|c|}
\hline \multirow{2}{*}{ RAZA } & DISPONIBLES & SINCRONIZADAS/ & TRANSFERIDAS/ & PREÑADAS/ & PREÑADAS/ \\
& DISPONIBLES (\%) & DISPONIBLES (\%) & TRANSFERIDAS (\%) & DISPONIBLES (\%) \\
\hline \multirow{2}{*}{ NORMANDO } & \multirow{2}{*}{88} & $\begin{array}{c}79 / 88 \\
(89.7)\end{array}$ & $\begin{array}{l}63 / 88 \\
(71.5)\end{array}$ & $\begin{array}{c}40 / 63 \\
(63.3)\end{array}$ & $\begin{array}{c}40 / 88 \\
(45.4)\end{array}$ \\
\hline \multirow{2}{*}{ HOLSTEIN } & \multirow{2}{*}{108} & $\begin{array}{c}106 / 108 \\
(98.1)\end{array}$ & $\begin{array}{c}90 / 108 \\
(83.3)\end{array}$ & $\begin{array}{c}50 / 90 \\
(55.5)\end{array}$ & $\begin{array}{c}50 / 108 \\
(50.9)\end{array}$ \\
\hline \multirow{2}{*}{ BRANGUS } & \multirow{2}{*}{102} & $97 / 102$ & $52 / 102$ & $23 / 52$ & $23 / 102$ \\
& & $(95)$ & $(50.9)$ & $(44.2)$ & $(22.5)$ \\
\hline \multirow{2}{*}{ Total } & 298 & $282 / 298$ & $205 / 298$ & $113 / 205$ & $113 / 298$ \\
& & $(94.6)$ & $(68.7)$ & $(55.1)$ & $(37.1)$ \\
\hline
\end{tabular}

Se presume que las bajas tasas de preñez, cuando se transfieren embriones de buena calidad, frescos o congelados, podrían estar relacionadas con la calidad del cuerpo lúteo (tamaño y concentración de progesterona), el grado de sincronía y la raza (cruzamiento) de la receptora. Para el reconocimiento materno embrionario, el embrión debe encontrar un medio uterino apropiado, influido por la progesterona (P4) lútea, ya que esta estimula la producción de una variedad de secreciones endometriales, como el MUC-1 (mucin glycoprotein-1), el lactógeno placentario y las osteopontinas, necesarias para el adecuado desarrollo de los embriones (4).

Por otra parte, la raza ejerce algún tipo de efecto con respecto al tamaño del cuerpo lúteo (por el tamaño del folículo ovulatorio y el número de células de la granulosa), lo cual puede estar ejerciendo un efecto positivo o negativo sobre las tasas de concepción, o la raza puede estar, a su vez, actuando directamente sobre las tasas de preñez, como lo reporta Olson (5); las diferencias reproductivas entre las razas europeas y las Bos indicus se presenta y tienen incidencia en los porcentajes de animales preñados, Pero según Portillo (6) el ganado mestizo (Brangus) presenta tiempos y niveles hormonales promedio, no obstante sería importante realizar estudio relacionados a determinar estos niveles entre razas puras y mestizas de este medio.

Velasco (7) argumenta que las novillas, normalmente, no se encuentran bajo estrés nutricional ni lactacional y presentan, en menor porcentaje, historial con problemas reproductivos, además de considerar el útero virgen más apropiado para recibir un embrión transferido. También consideran que así como en la inseminación artificial, en la transferencia embrionaria se obtiene mayor porcentaje de preñez en novillas que en vacas, debido a la influencia que posiblemente ejerce la suplementación proteica, que puede generar descensos en el $\mathrm{pH}$ uterino, lo cual, en combinación con bajos niveles de P4, crea un ambiente hostil para el embrión.

Preñadas/Transferidas. Según los resultados, se observa que la raza Normando presentó un porcentaje del $63.3 \%$ de novillas preñadas, seguida de la raza Holstein, con $55.55 \%$, mientras que la raza Brangus presentó un $44.23 \%$. En la Figura 1 se presenta el porcentaje de preñadas/ transferidas de cada raza evaluada. El análisis chi cuadrado nos indica que entre Normando y Holstein no hay diferencia estadística significativa $(p \geq 0.322)$, que entre Holstein y Brangus sí hay 
diferencia estadística significativa $(p \geq 0.049)$ y que entre Normando y Brangus sí hay diferencia estadística significativa $(\mathrm{p} \leq 0.036)$.

Las dos razas puras (Normando y Holstein) están bien adaptadas al clima frío y a la topografía quebrada, y la raza Brangus, que es un cruce entre Brahman y Angus, es más adaptable a climas intermedios. Según Donaldson (8), las razas cruzadas se prefieren más que las puras por sus mejores resultados; afirmación que no coincide con este estudio. Por otra parte, Rodríguez (9) plantea que una buena receptora, en lo posible, debe ser tranquila, y en la fase de experimentación se evidenció que las razas Normando y Holstein son animales tranquilos y de fácil manejo y adaptación al medio predominante frío $\left(12{ }^{\circ} \mathrm{C}\right.$ y 2740 msnm, en promedio, de la finca), y que, por el contrario, la raza Brangus es mucho más nerviosa y de difícil manejo. Esto no significa que los resultados obtenidos por la raza Brangus sean malos, ya que estudios realizados en Puerto Araujo (Santander), por Ariza (10), reportan un $33.4 \%$ de animales preñados, porcentaje menor que el obtenido en este estudio por la misma raza Brangus.

Restrepo (11) ha definido en el bovino los atributos y las características morfológicas y fisiológicas que reflejan la adaptación al medioambiente, como selección por eficiencia o por actitud funcional, cuya base principal es la apreciación de signos externos e internos que nos indican fertilidad.

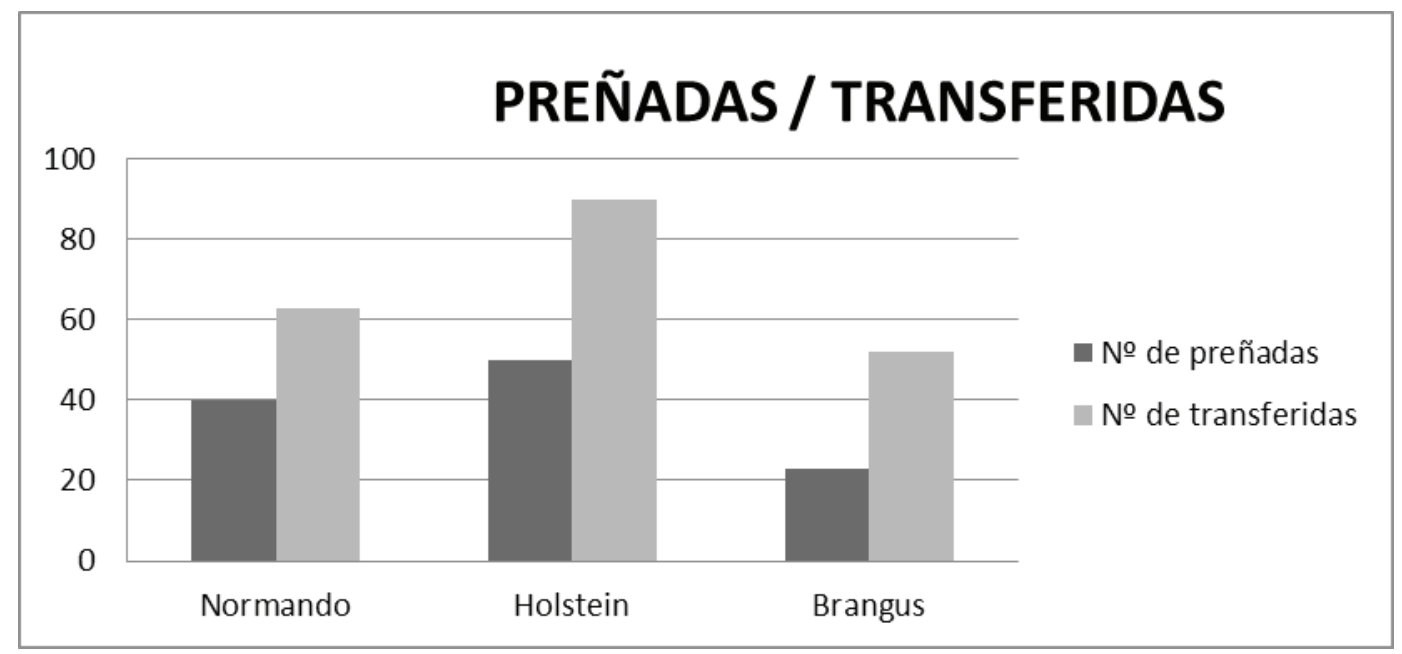

Figura 1. Preñadas/Transferidas

Preñadas/Disponibles. Existe diferencia estadística significativa $(p \leq 0.05)$ entre Normando y Brangus, al igual que entre Holstein y Brangus, y no se presenta diferencia estadística significativa entre Normando y Holstein $(p \leq 0.05)$. La Figura 2 presenta el porcentaje preñadas/disponibles de cada raza. Los resultados obtenidos indican que se requiere de un número potencial de receptoras muy alto, lo que incrementa el precio de sostenimiento de las receptoras.

Existe evidencia de que la procedencia de la receptora afecta significativamente el porcentaje de preñeces (8); así mismo, la disponibilidad y la adaptación al medio, en términos de alimentación, enfermedades y cambios climáticos, son posiblemente los factores que determinan la elección de la receptora.

Según Pietro et al. (2005), utilizar novillas de la misma finca o región de donde se pretende realizar la transferencia puede incrementar del $10 \%$ al $15 \%$ la cantidad de preñadas, en comparación con animales de otras regiones o recién traídas; lo anterior se evidencia en las razas Normando y Holstein, procedentes de regiones similares a las condiciones ambientales del lugar de experimentación. 


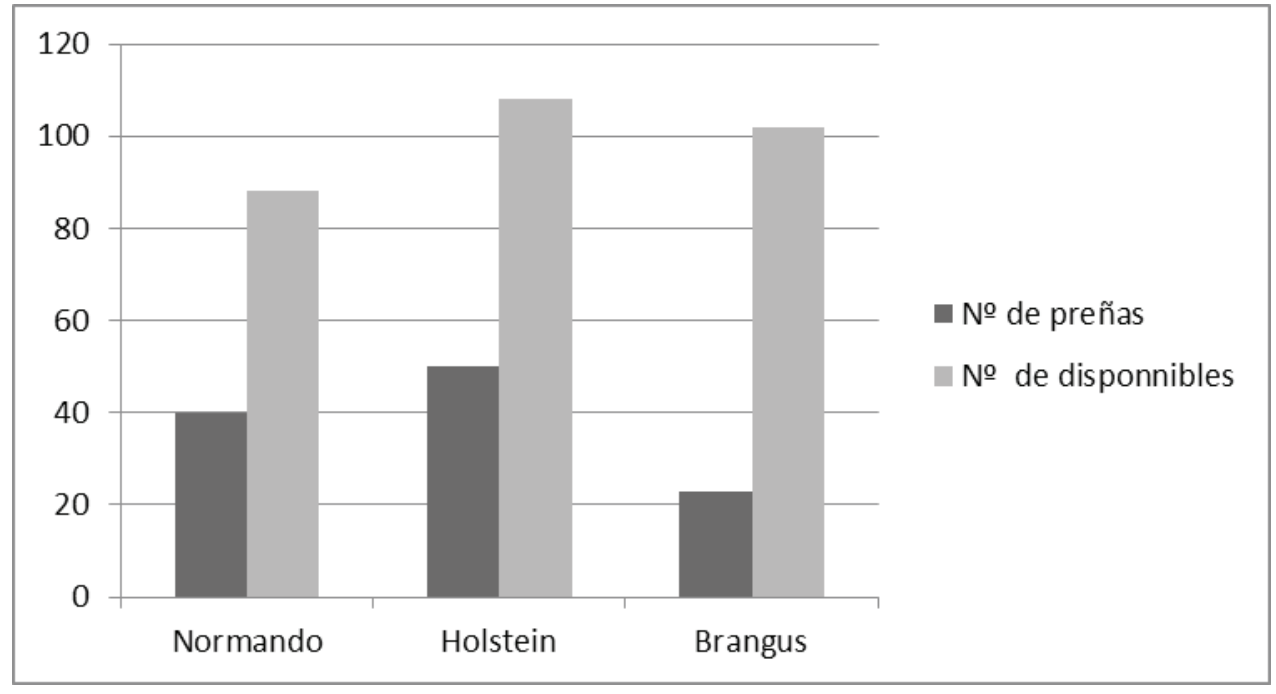

Figura 2. Preñadas/Disponibles

Promedio y desviación estándar del peso y la condición corporal (CC) por raza.La condición corporal y el contenido energético de las receptoras son muy importantes; las razas en estudio registraron un promedio de la CC y el peso como se muestra en la Tabla 2; esta presenta el promedio y la deviación estándar del peso y la CC en escala de 1 (muy flaca) a 5 (muy gorda), valores que están dentro de los recomendados por Pettit (11); esto quiere decir que, posiblemente, la nutrición no fue determinante en el resultado del porcentaje de novillas preñadas de cada raza, ya que todas estuvieron bajo las mismas condiciones nutricionales.

Tabla II. Promedio y desviación estándar de peso y CC por raza

\begin{tabular}{|c|c|c|c|}
\hline RAZA & NORMANDO & HOLSTEIN & BRANGUS \\
\hline $\begin{array}{c}\text { PROMEDIO DE } \\
\text { PESO }\end{array}$ & 379.7 & 366.2 & 370 \\
\hline $\begin{array}{c}\text { DESVIACIÓN } \\
\text { ESTÁNDAR DEL } \\
\text { PESO }\end{array}$ & 16.7 & 19.6 & 16.76 \\
\hline PROMEDIO CC & 3.1 & 2.97 & 2.75 \\
\hline $\begin{array}{c}\text { DESVIACIÓN } \\
\text { ESTÁNDAR DE } \\
\text { LA CC }\end{array}$ & 0.3 & 0.38 & 0.38 \\
\hline
\end{tabular}

\section{CONCLUSIONES}

Se observó que la raza Normando presentó el mayor porcentaje de novillas preñadas $(63.3 \%)$, seguida de la raza Holstein $(55.55 \%$ ) y, por último, de la raza Brangus (44.23\%). Es factible que las tasas de preñez presentadas estén relacionadas con la calidad del cuerpo lúteo (tamaño y concentración de progesterona), el grado de sincronía y la raza (cruzamiento) de la receptora. En el estudio se descarta que el aspecto nutricional influya en la tasa de preñez presentada en cada una de las razas evaluadas.

\section{REFERENCIAS}

(1) Hansen J. Preparación de embriones producidos in vitro para transferir a receptoras. Departamento de Ciencia Animal y Clínica de Grandes Especies, Universidad de Florida. (en línea), 2007 (fecha de consulta: 13 de febrero de 2013). Disponible en http//www.jus.fass.org.

(2) Gómez CJ. Transferencia de embriones experiencias en Colombia. Memorias del Congreso Internacional de Reproducción Bovina (Intervet), Bogotá, 2005, p.155-158.

(3) De Luca LJ. Niveles plasmáticos de progesterona en receptoras de Embriones Congelados Determinados por Test de elisa. 2007 (fecha de consulta: 11 de marzo de 2013). Disponible en http//www.produccionanimal.com.ar 
(4) Demmers KJ, Derecka K, Flint A. Trophoblast Interferon and Pregnancy. Reproduction 2001; 8(1): 46-53.

(5) Olson T. Composite Breeds in the Tropics. Thesis, Animal Science Department, University of Florida, 2005.

(6) Portillo M. Fisiología reproductiva y diferencias reproductivas entre el ganado europeo y cebú. Manual de ganadería doble propósito. 2005.

(7) Velasco F. Análisis retrospectivo de los resultados obtenidos en la Unidad de Transferencia de Embriones Amelis, en la raza Normando durante el periodo 2000-2007. Universidad Pedagógica y Tecnológica de Colombia. 2009.

(8) Donaldson L.E. Recipients as a source of variation in cattle embryo transfer. Theriogenology 2005; 23: 188-196.
(9) Rodríguez J.M, Giraldo C. Análisis multifactorial de las tasas de preñez en programas de transferencia de embriones en Colombia. Rev. MVZ Córdoba 2007; 12(2): 978-984.

(10)Ariza L. Evolución retrospectiva de la tasa de preñez obtenida por transferencia de embriones en diferentes cruces bovinos en el municipio de Puerto Araujo (Santander). Colombia. Revista electrónica de veterinaria REDVET, 2006; 7 (4): 345-354.

(11)Restrepo J. Multicruces, Proyecto técnicamente viable y económicamente rentable. 2004.

(12)Broadbent P.J, Stewart M.D. Recipient Management and Embryo Transfer. Theriogenology 1999; 17:123-139.

(13)Pettit G. Commercial freezing of bovine in glass ampules. Theriogenology 2006; 24: 3-16. 